Cátedras de radio en contingencia por la pandemia COVID-19. Relevamiento federal de las estrategias pedagógicas desarrolladas en tiempos de no presencialidad

María Elena Beneitez, Matías Delménico, Ana Laura Gratti, Bibiana Parlatore

Question/Cuestión, Nro.69, Vol.3, diciembre 2021

ISSN: $1669-6581$

URL de la Revista: https://perio.unlp.edu.ar/ojs/index.php/question/

IICom -FPyCS -UNLP

DOI: https//doi.org/10.24215/16696581e588

\title{
Cátedras de radio en contingencia por la pandemia COVID-19
}

\section{Relevamiento federal de las estrategias pedagógicas desarrolladas en tiempos de no presencialidad}

Radio chairs in contingency due to the Covid-19 pandemic

Federal survey of pedagogical strategies developed in times of non-presence

\section{María Elena Beneitez}

Licenciada en Comunicación Social con Orientación en Periodismo (FPyCS-UNLP) y Locutora Nacional (FPyCS-ISER). Profesora Adjunta del Taller de Producción Radiofónica III, Cátedra 1. Extensionista y Docente investigadora de la FPyCS-UNLP 


\section{Matías Delménico}

Licenciado y Profesor en Comunicación Social con Orientación en Periodismo (FPyCS-

UNLP).Especialista en Prácticas, medios y ámbitos educativos y Docente del Taller de Estrategias de Comunicación Sonora y Radial, cátedra 1. Extensionista y Docente investigador de la FPyCS-UNLP. Docente en nivel secundario superior en la provincia de Buenos Aires.

Argentina

matiasdelmenico@gmail.com

\section{Ana Laura Gratti}

Profesora y Licenciada en Comunicación Social con orientación en Periodismo(FPyCS-UNLP). Docente del Taller de Estrategias de Estrategias de Comunicación Sonora y Radial, cátedra 1. Docente en nivel secundario y superior de la provincia de Buenos Aires.

Argentina analauragratti@gmail.com

\section{Bibiana Parlatore}

Licenciada en Comunicación Social con Orientación en Periodismo (FPyCS-UNLP). Docente del Taller de Estrategias de Comunicación Sonora y Radial, cátedra 1. Productora de contenidos y conductora en Radio Universidad Nacional de La Plata.

Argentina bibianaparlatore@gmail.com

Edición sonora: Equipo de operadores técnicos de la FPyCS-UNLP

Diseño de imágenes: Federico Etcheverry 


\section{Resumen}

Se trata de un trabajo desarrollado en el Instituto de Investigaciones en Comunicación (IICom) de la Facultad de Periodismo y Comunicación Social de la Universidad de La Plata y que fue encabezado por docentes del Taller de Estrategias de Comunicación Sonora y Radial, Cátedra 1 durante un año, entre agosto 2020 y agosto 2021.

La investigación surge a partir de la pregunta ¿qué estrategias pusieron en práctica las cátedras de radio de las universidades nacionales para sostener la continuidad pedagógica durante el periodo de enseñanza virtual?

El trabajo abarca, por un lado, un relevamiento federal, que contempla las posibilidades tecnológicas, artísticas, estéticas y formas de adaptación al contexto de enseñanza durante la no presencialidad.

Y por el otro, el desarrollo de entrevistas para recuperar los relatos y habilita la posibilidad de que las docentes y los docentes tomen la palabra y describan sus formas de trabajo, de acuerdo con las condiciones propias de cada provincia, región, unidad académica e interlocutoras e interlocutores.

Palabras clave: radio; lenguaje sonoro; educación virtual; universidad; pandemia; enseñanza; tecnología; virtualidad

\section{Abstract}

This is a work developed at the Institute for Research in Communication (IICom) of the Faculty of Journalism and Social Communication of the University of La Plata and which was led by teachers from the Workshop on Sound and Radio Communication Strategies, Chair 1 during a year, between August 2020 and August 2021.

\footnotetext{
IICom (Instituto de Investigaciones en Comunicación) 
The research arises from the question, what strategies did the radio chairs of national universities put into practice to sustain pedagogical continuity during the virtual teaching period?

The work includes, on the one hand, a federal survey, which contemplates the technological, artistic, aesthetic possibilities and forms of adaptation to the teaching context during non-presence.

And on the other, the development of interviews to recover the stories and enables the possibility for teachers to speak and describe their ways of working, according to the conditions of each province, region, academic unit and interlocutors.

Keywords: radio; sound language; virtual education; university; pandemic; teaching; technology; virtuality

El 2020, año del Bicentenario del General Manuel Belgrano en nuestra Universidad Nacional de La Plata, quedó teñido con un hecho tan extraordinario que quedará en la memoria de quienes somos parte de esta Casa como el año de la Pandemia COVID-19.

La resolución №667 de la Universidad Nacional de La Plata, del 15 de marzo de 2020, dispone la "no realización de instancias áulicas presenciales de enseñanza" en el ámbito de esta casa de estudios. Las cátedras de cada una de las unidades académicas debimos modificar nuestras estrategias de enseñanza para poder desarrollarlas de manera virtual.

Desde el Taller de Estrategias de Comunicación Sonora y Radial, cátedra 1, de la Facultad de Periodismo y Comunicación Social nos propusimos investigar, conocer y describir las estrategias que pusieron en práctica las cátedras de radio de las universidades nacionales para sostener la continuidad pedagógica durante el periodo de enseñanza virtual.

El trabajo "Cátedras de radio en contingencia por la pandemia Covid-19. Relevamiento federal de las estrategias pedagógicas desarrolladas por las cátedras de radio en Universidades Nacionales en tiempos de no presencialidad" recupera las estrategias didácticas, 
en general, y procesos educativos, en particular, desarrolladas por docentes de radio de 17 UUNN durante la contingencia. La investigación -en proceso de análisis de datos y edición de materiales sonoros- contempla las posibilidades tecnológicas, artísticas, estéticas y formas de adaptación al contexto. El relevamiento se extendió hasta mediados de 2021.

\section{Datos técnicos}

El relevamiento obtuvo 27 respuestas de 17 universidades nacionales, distribuidas a lo largo y ancho de la geografía nacional. Se trata de las Universidades Nacionales de La Plata, Rosario, Entre Ríos, Tucumán, Patagonia Austral Unidad Académica Río Gallegos, Salta, La Matanza, La Pampa, Moreno, Río Cuarto, Jujuy, Córdoba, Avellaneda, Comahue, Buenos Aires, General Sarmiento y La Rioja.

Las encuestas fueron desarrolladas a través del software de administración de encuestas "formulario de Google" y enviadas a través de correo electrónico durante el mes de noviembre de 2020 y el mes de agosto de 2021.

La metodología de investigación bajo la cual se desarrolló el trabajo es el Método de Comparación Constante (MCC). A su vez, las técnicas específicas fueron: la encuesta, como procedimiento que posibilita la recopilación de datos mediante un cuestionario predeterminado; y las entrevistas telefónicas, para indagar y ampliar a partir de la escucha activa y metódica de las experiencias seleccionadas para la producción de la serie de podcast.

\section{Enseñar radio en la no presencialidad}

Las cátedras de radio sobre las cuales se desarrolló el relevamiento pertenecen a las carreras de Licenciatura en Comunicación Social, Periodismo y una tecnicatura específica en comunicación. A su vez, el 25,9\% de esas materias se dictan en el primer año de la carrera; el $40,7 \%$ en el segundo; el $22,2 \%$ en tercero; el 7,4 en cuarto y el 3,7\% en quinto año. 
La encuesta preliminar, que nos permitió seleccionar algunas experiencias en las cuales profundizar y contrastar, estableció que una mayoría contundente pudo adaptarse al contexto de contingencia y sostener las prácticas radiales y sonoras (96,3\%). La única cátedra que indicó que no pudo adaptarse (3,7\%), adujo como motivo "la falta de estudio de radio".

Para profundizar en las modalidades de trabajo habitual (previo a la pandemia) de las propuestas curriculares, se indagó en los espacios en que se realizan o difunden las prácticas.

Hacia el interior de la cátedra: $40,7 \%$

Emisoras universitarias: $22,2 \%$

Emisoras comunitarias: $14,8 \%$

Plataformas de sonido: $14,8 \%$

En redes: $3,7 \%$

Sitios Web: $3,7 \%$

Respecto al contexto de pandemia propiamente dicho, el $81,5 \%$ indicó que pudo sostener las prácticas de producción radial y/o sonora, mientras que el 18,5\% indicó lo contrario. En ese sentido, las estrategias señaladas fueron: "enlatamos producciones" (63\%), "realizamos producciones virtuales en vivo $(11,1 \%)$, "realizamos producciones virtuales símil vivo para emitir en diferido" (3,7\%). El resto combinó algunas de las opciones anteriores.

Las plataformas en las que desarrollaron las clases fueron las siguientes: Zoom: 55,6\% Meet: $44,4 \%$

Campus virtual de la Universidad: $3 \%$

Web/Blog de cátedra: 25,9\%

Aulas virtuales: $18,5 \%$

Youtube: $14,8 \%$ 
Otras: $14,8 \%$

A su vez, y además de las plataformas, el 100\% afirmó utilizar otras formas de contacto a las mencionadas y se distribuyen de la siguiente manera:

WhatsApp: $29,6 \%$

E-mail: $22,2 \%$

Facebook: 14,8\%

Mensajería propia de la facultad: 7,4\%

Classroom: $7,4 \%$

Combinaron dos o más opciones: $18,5 \%$

Respecto de las estrategias elegidas para el desarrollo de las clases teóricas sobresalen las reuniones sincrónicas, con un $55,6 \%$ de respuestas.

El 44,4\% optó por otras estrategias ofrecidas en la encuesta sin sobresalir ninguna de ellas y entre las que se encontraban la utilización de Videos, Audios, PDF, Mix de clases sincrónicas y asincrónicas, Podcast; Videos propios y ajenos, y combinación de opciones.

Con relación a la utilización de plataformas para alojar contenidos sonoros, el 59,3\% indicó que habilitaron esos espacios como parte de las estrategias de tarea pedagógica. Entre ellas, sobresalen Ivoox (26,3\%); SoundCloud (15,8\%). También fueron mencionadas: RSS, aula virtual, Padlet, Sitio de la Universidad, Blog, Classroom, Google Podcast, Spreaker, App Podcast, Drive.

Cabe señalar que en la encuesta fueron ofrecidas como opciones las plataformas Spotify, Mixcloud y Spreaker que no fueron seleccionadas por ninguna cátedra de radio para alojar sonido.

Por último, un dato importante que relevó el trabajo es la relación entre la deserción en relación a la modalidad de cursada. En ese sentido, un $44,4 \%$ de los entrevistados y las 
entrevistadas afirmó detectar deserción a causa de la virtualidad, en tanto que un 40,7\% se expresó por la negativa. El restante $14,8 \%$ afirmó no poder detectar la influencia de la modalidad en la deserción de los/as estudiantes.

\section{Caracterización de las propuestas pedagógicas}

En el marco de este relevamiento federal de estrategias utilizadas por las cátedras de radio y de producción sonora de las carreras de Comunicación de Universidades Nacionales en tiempos de contingencia producto de la pandemia por el COVID-19, nos centraremos aquí en la caracterización de las estrategias didácticas desarrolladas para la no presencialidad durante 2020-2021.

Con el objetivo de sostener la continuidad pedagógica, las cátedras debieron redefinir los contenidos prioritarios de los programas, reformular diferentes aspectos de las clases, formas de trabajo, propuestas de evaluación y acreditación de saberes, y capacitarse en el devenir de la pandemia, atendiendo a las trayectorias formativas de los estudiantes y las estudiantes.

\section{Capacitación y aprendizajes}

En las experiencias que compartieron los docentes y las docentes de las diferentes Universidades, se evidencia la combinación de clases sincrónicas y asincrónicas, con estrategias didácticas específicas para cada caso.

Para llevar adelante el desarrollo de los talleres durante la virtualidad, resultó central apostar a la capacitación en recursos tecnológicos. Por un lado, el uso de herramientas para videoconferencias, como Meet o Zoom, posibilitó la sincronía de los intercambios, y las prácticas sonoras "en vivo". Además, en muchos casos, fue necesario aprender a utilizar plataformas, como aulas virtuales o webs de cátedra en los sitios desarrollados por las Facultades. Ya sea de forma autodidacta, con tutoriales, o colectivamente, con el apoyo entre 
compañeros y compañeras de Cátedra, o a través de dispositivos de capacitación habilitados por las propias universidades nacionales se fue haciendo camino al andar.

Lo interesante es que estos procesos formativos de los docentes y las docentes, surgidos en la urgencia y ante la necesidad de sostener las cursadas en pleno aislamiento social preventivo y obligatorio, hoy constituyen un acervo que seguramente transforme y enriquezca las prácticas docentes en la post-pandemia: "Salimos fortalecidos en el manejo de nuevas herramientas y recursos que pensamos llevar a la presencialidad".

Sin embargo, el manejo tecnológico fue uno de muchos otros desafíos que debieron afrontar los docentes y las docentes de las cátedras de radio y producción sonora: "hubo que replantear la producción de los contenidos, y las formas conocidas". Así, además de los contenidos conceptuales, debieron repensar el manejo de los tiempos de las clases, la periodicidad de los encuentros, las propuestas de trabajo individuales y grupales, los requisitos para las producciones sonoras, entre otras cuestiones.

Un punto de contacto central, en las experiencias de las cátedras, es la modalidad de "taller". El eje vertebrador de las diferentes materias continúa en línea con aquello que esta metodología implica: el aprender haciendo, con otros y otras. Lo experiencial, constitutivo de la radio y del universo sonoro, se traduce en el trabajo en equipo, colaborativo, en proceso. Esto continúa teniendo protagonismo en los modos de hacer -y aprender a hacer- radio y contenidos sonoros.

A continuación, compartiremos algunas de las estrategias que fueron construyendo y reconstruyendo, a lo largo de esta pandemia y desde la virtualidad, los diferentes equipos de cátedra de radio de las universidades nacionales que forman parte de este estudio.

\section{Clases sincrónicas}

Un aspecto fundamental tiene que ver con la planificación de los tiempos de las instancias sincrónicas. Resultó evidente que los tiempos y la dinámica de las clases presenciales no podían replicarse en la virtualidad. Este aprendizaje de los equipos de cátedra,

\footnotetext{
IICom (Instituto de Investigaciones en Comunicación) 
que en muchos casos se inició desde la "improvisación", se plasmó luego en estrategias concretas: "acortar las duraciones de las prácticas", "administrar el uso de la videoconferencia", reducir los tiempos de las clases semanales a la mitad o menos, por ejemplo. Incluso, algunos equipos modificaron la regularidad de los encuentros. A su vez, habilitaron espacios más personalizados: encuentros semanales de consulta fijos o reuniones por turnos, a modo de tutorías, para complementar las clases.

Muchas de las cátedras se caracterizan por realizar prácticas en estudio de radio y programas en emisoras universitarias o locales, que se vieron interrumpidos por el aislamiento obligatorio. Por lo tanto, se generaron programas en vivo en las clases sincrónicas, a través de Meet o Zoom, y también se fortaleció el desarrollo de producciones enlatadas, como microproducciones y podcasts. Algunos y algunas estudiantes trabajaron con Wetransfer, por ejemplo.

Otro recurso interesante consistió en "abrir el juego con docentes, autores, colegas que como 'invitados' participaron de las clases desde sus experiencias y abordajes", además de entrevistados acerca de diferentes temáticas, que participaron de las clases sincrónicas .

\section{Clases asincrónicas}

A través de las plataformas de las Universidades, Google Drive o las webs de cátedra en las páginas de las Facultades, y la utilización de correo electrónico o grupos de Whatsapp, los diferentes equipos de cátedra generaron distintas posibilidades de trabajo "domiciliarios", individuales y grupales, para las instancias no presenciales.

En general, las plataformas sirvieron de base para la socialización "de los materiales teóricos y ejemplos sonoros". En algunos casos, se trató de bibliografía específica y de propuestas teóricas elaboradas para la ocasión, a modo de documentos, fichas o apuntes de cátedra escritos, aunque también "se combinaron con producciones de cátedra en audio, en formato podcast". Esto constituyó un verdadero trabajo de producción de contenidos desde las cátedras, de materiales educativos especialmente pensados y elaborados teniendo en cuenta el contexto. 
Respecto de los materiales teóricos, la experiencia mostró que ya no era posible trabajar de la misma manera que antes de la pandemia, por lo que reconocieron que fue necesario hacer una selección y jerarquización de textos y, en algunos casos, "dosificar la bibliografía", realizando un recorte de los ejes considerados fundamentales, profundizando, e incluso incursionando, en la curaduría de contenidos digitales.

Además de la lectura de la bibliografía y las propuestas de trabajos prácticos, se intensificó "la tarea de análisis y reflexión acerca de la comunicación radiofónica", ya que "disminuyeron notablemente las posibilidades de realización práctica", al menos a inicios de la pandemia.

Se alentó "la escucha de radio y de podcast" y su análisis. Se lograron "sostener foros de intercambio conceptual y teórico" posibilitando "un intercambio reflexivo y crítico sobre el análisis de las prácticas".

Algunas experiencias destacaron el trabajo "en escala", proponiendo diferentes instancias, a modo de proceso, que finalizaron con las producciones sonoras: "Primero grabaciones con lectura de texto, luego con sonidos a través de edición de audio, para culminar con informes periodísticos donde se aplicó lo aprendido".

\section{Las producciones sonoras}

La combinación de las diversas estrategias sincrónicas y asincrónicas de los equipos de cátedra tuvo como eje central el desarrollo de piezas sonoras, principalmente grupales. Desde una perspectiva mucho más anclada en los procesos que en la excelencia técnica como resultado, se apostó a un recorrido formativo que le permitiera a los y las estudiantes transitar los diferentes roles e instancias del proceso de producción. La experiencia fue "innovadora, por primera vez les estudiantes desarrollaron todo el proceso de producción de materiales sonoros, desde su concepción, grabación, edición y publicación en agregadores o plataformas de streaming de audio". 
Además de la realización de programas de radio y producciones en diferentes géneros y formatos, como entrevistas, comentarios, boletines informativos, informes periodísticos, entre otros, el protagonista de las propuestas fue el podcast: "Para esta situación de excepcionalidad se priorizó la idea central del Módulo de Radio que se expresa en la frase "Hacer Radio Haciendo Radio. Por tal razón, las producciones consistieron en dos series de podcast referidos a los 100 Años de la Radio".

\section{Vínculo con estudiantes y acompañamiento pedagógico}

En la virtualidad, además de la comunicación entre estudiantes y docentes a través del correo electrónico, grupos de Whatsapp, avisos, o correo interno de las plataformas, emergieron los foros como un espacio de socialización e intercambio. El diálogo también fue posible en las clases sincrónicas y en los espacios de tutorías, de forma más personalizada: "Optamos por continuar intensivamente la atención de cada equipo de trabajo para que las producciones que cada año se realizan no corrieran peligro. Fue difícil, pero pudimos lograrlo. El resultado de las producciones ha sido óptimo. Se podría decir que prácticamente al igual que un año normal. Aunque, claramente, para ello hizo falta redoblar esfuerzos entre todas las partes (docentes y estudiantes)".

Es evidente que la construcción de la comunicación y los lazos entre estudiantes y docentes ha sido "de contingencia, haciendo eje en sostener el vínculo con estudiantes que ponían su esfuerzo en hacer frente a problemas de conectividad, organización familiar y laboral", por ejemplo.

\section{Valoración de las estrategias}

En relación con las estrategias sincrónicas y asincrónicas construidas en la virtualidad, en este escenario de contingencia, los diferentes equipos de cátedra valoraron positivamente los resultados de su puesta en juego, producto de la creatividad, del trabajo en equipo, que se inició casi a tientas: "Las estrategias implementadas se basaron (en un principio) en el ensayo y

\footnotetext{
IICom (Instituto de Investigaciones en Comunicación) 
el error. En todo momento se trató de resguardar los fundamentos de la perspectiva pedagógica basada en los Ejes Hablar de Radio, Escuchar Radio y Hacer Radio".

La revisión de la propia práctica y las múltiples reformulaciones de las propuestas que se fueron generando al interior de los equipos de trabajo, hoy permiten una reflexión mayor del proceso: "Fueron prácticas urgentes, de excepción; que garantizaron el dictado de contenidos. Se dieron dos momentos: uno de simultaneidad entre el dictado de clases y la adaptación a la nueva instancia, pensando con el conjunto del Taller los modos, apoyados por la Facultad y la Dirección de Educación a Distancia de la UNLP; y otro, de consolidación de la estrategia, con niveles de negociación con los estudiantes".

En líneas generales, las estrategias didácticas desarrolladas han permitido lograr los propósitos fundamentales de las materias de radio y producción sonora y son consideradas "positivas", "efectivas, dado el contexto". Las estrategias fueron "novedosas", "esperadas y necesarias para docentes y estudiantes; fueron deaprendizaje e innovación", "experimentales pero satisfactorias por los resultados obtenidos. Los estudiantes se adaptaron rápidamente".

También se las considera "variadas, acordes a cada grupo de comisión y de los recursos de les estudiantes", lo que da cuenta de los procesos de reconocimiento de las realidades de los diferentes grupos, sus contextos y características: "Nos obligó a crear dinámicas particulares para el soporte utilizado". Estos aspectos se tuvieron en cuenta en gran medida para prealimentar las prácticas, y posibilitar la continuidad pedagógica en una coyuntura tan particular.

En algunos casos se considera a las clases sincrónicas y asincrónicas como "limitadas" o "básicas", desde el punto de vista técnico, ya que, por ejemplo, "la edición vía Zoom se complejizó" con la virtualidad. Así, emerge la necesidad de presencialidad, de una comunicación interpersonal que converja en tiempo y espacio, e implique poner el cuerpo: " $A l$ margen de que los contenidos se dieron, la imposibilidad de compartir -por ejemplo- en un mismo espacio la escucha de alguna producción radial redujo al mínimo nuestras posibilidades pedagógicas. Para nosotros, es fundamental ver cómo reaccionan los alumnos frente a un material sonoro determinado. Eso es imposible de lograr cuando damos clases virtuales frente a pantallas sin cámaras activadas. El resultado final, a pesar de ello, no fue malo. Las 
producciones individuales fueron satisfactorias, aunque presuponemos que el compromiso con la asignatura estuvo lejos de ser el mismo que obtuvimos en años anteriores".

Por otra parte, se destaca que se trató de una tarea "desafiante y compleja en términos de adaptación pedagógica", lo que evidentemente constituye un proceso formativo significativo para los equipos de cátedra, que ha permitido construir estrategias que podrían capitalizarse incluso para la vuelta a la presencialidad: "Las definiría como prácticas, lúdicas y eficientes. Así lo percibieron los propios alumnos, destinatarios de la propuesta. Más allá de que son estrategias pensadas como una salida temporal a la emergencia, pensamos que algunas fueron tan satisfactorias, que podrían continuar en la 'nueva normalidad"'.

\section{Recuperar las voces}

El estudio de cátedra pone en diálogo las formas de trabajo desarrolladas durante el periodo de enseñanza virtual en cátedras de radio de universidades nacionales y reconoce, a partir de ello, una multiplicidad de experiencias en un momento histórico desafiante para la docencia universitaria en general, y para el medio radiofónico y sonoro, en particular.

La investigación apunta, a su vez, a recuperar los relatos, habilitando la posibilidad de que sus docentes tomen la palabra y describan sus formas de trabajo, de acuerdo con las condiciones propias de cada universidad nacional, provincia, región, unidad académica e interlocutoras e interlocutores.

De este modo, la sistematización de las experiencias detallada deriva -a su vez- en la selección y recorte de algunas prácticas significativas para focalizar, a través de la realización de entrevistas, de recursos y de producciones sonoras propias de las experiencias seleccionadas, para la producción de podcast que configurarán la materialización final del trabajo. 
Avance sonoro del trabajo "Cátedras de radio en contingencia por la pandemia Covid19. Relevamiento federal de las estrategias pedagógicas desarrolladas por las cátedras de radio en Universidades Nacionales en tiempos de no presencialidad".

\section{Anexo}

"Cátedras de radio en contingencia por la pandemia Covid-19. Relevamiento federal de las estrategias pedagógicas desarrolladas por las cátedras de radio en Universidades Nacionales en tiempos de no presencialidad"

Cátedras de radio en contingencia por la pandemia Covid-19

Relevamiento federal de las estrategias pedagógicas

desarrolladas por las cátedras de radio en

Universidades Nacionales en tiempos de no presencialidad.

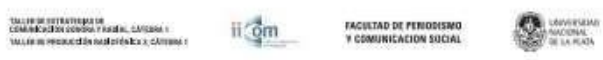

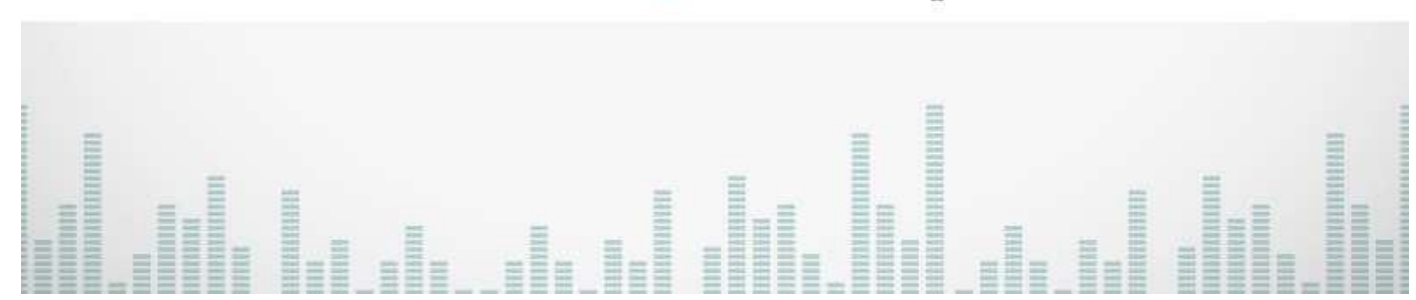


Question/Cuestión, Vol.3, N 70

Diciembre 2021

ISSN 1669-6581
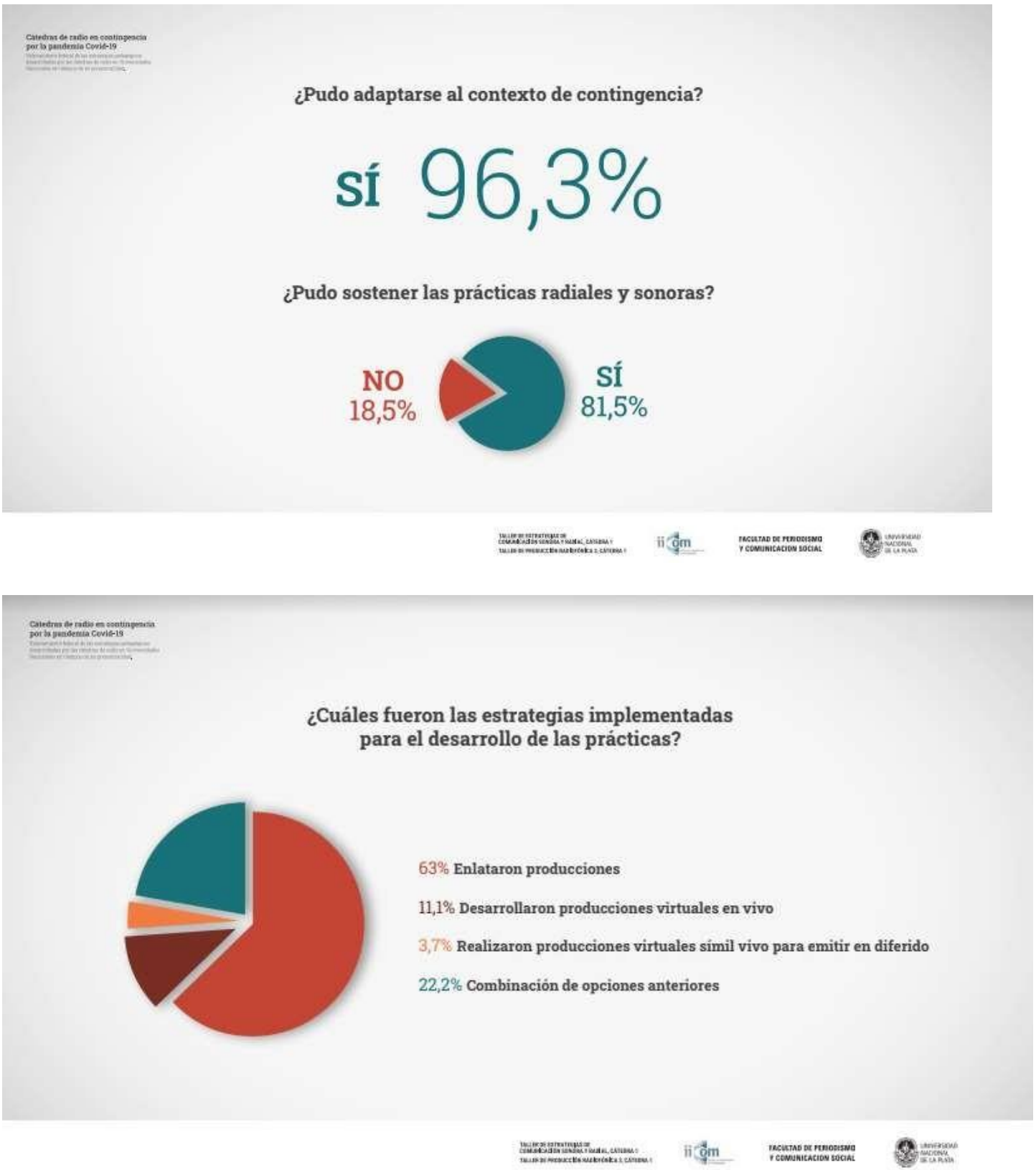

IICom (Instituto de Investigaciones en Comunicación)

Facultad de Periodismo y Comunicación Social

Universidad Nacional de La Plata 
Question/Cuestión, Vol.3, № 70

Diciembre 2021

ISSN 1669-6581

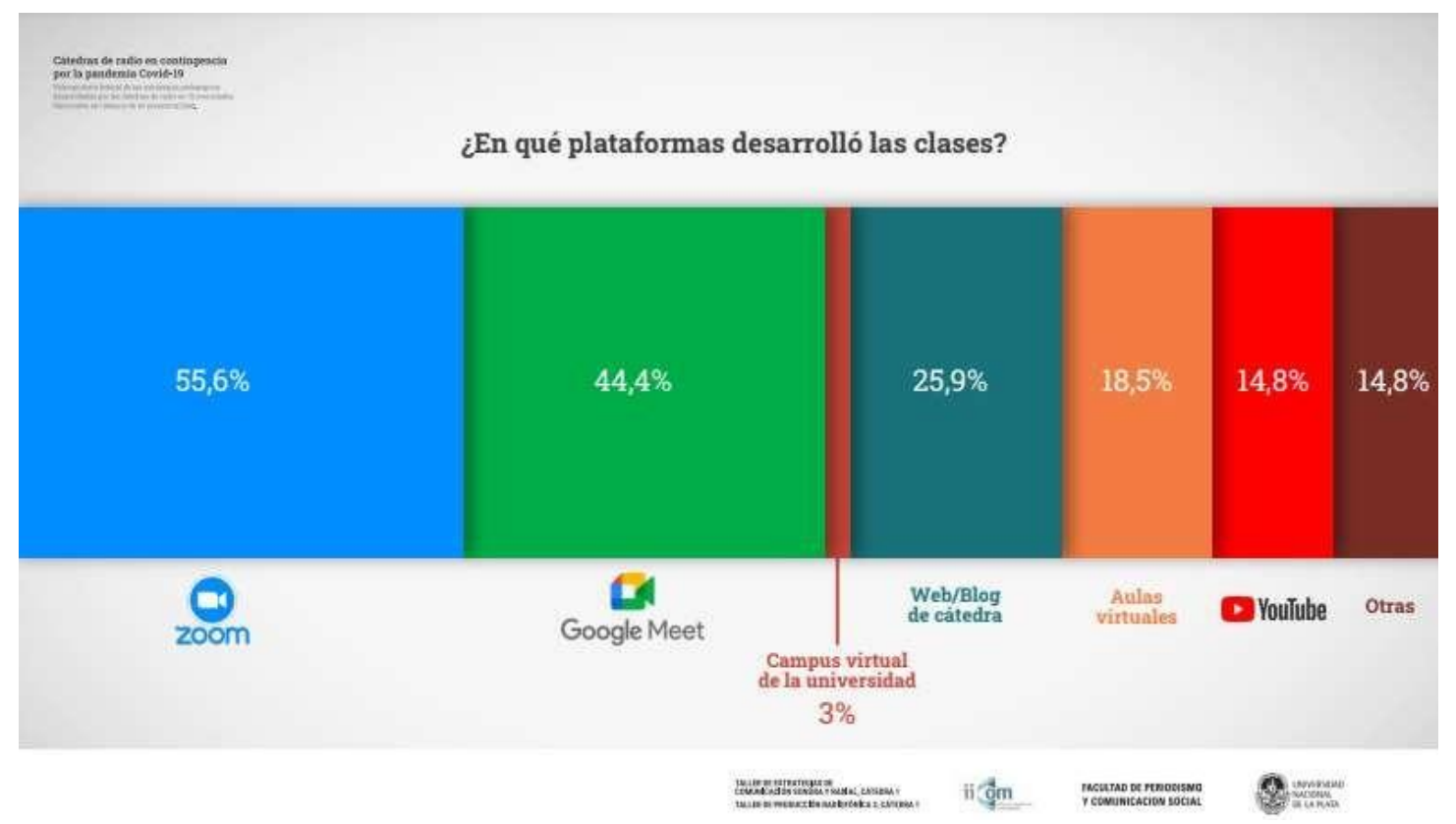

¿Qué estrategias de trabajo pedagógico utilizó?

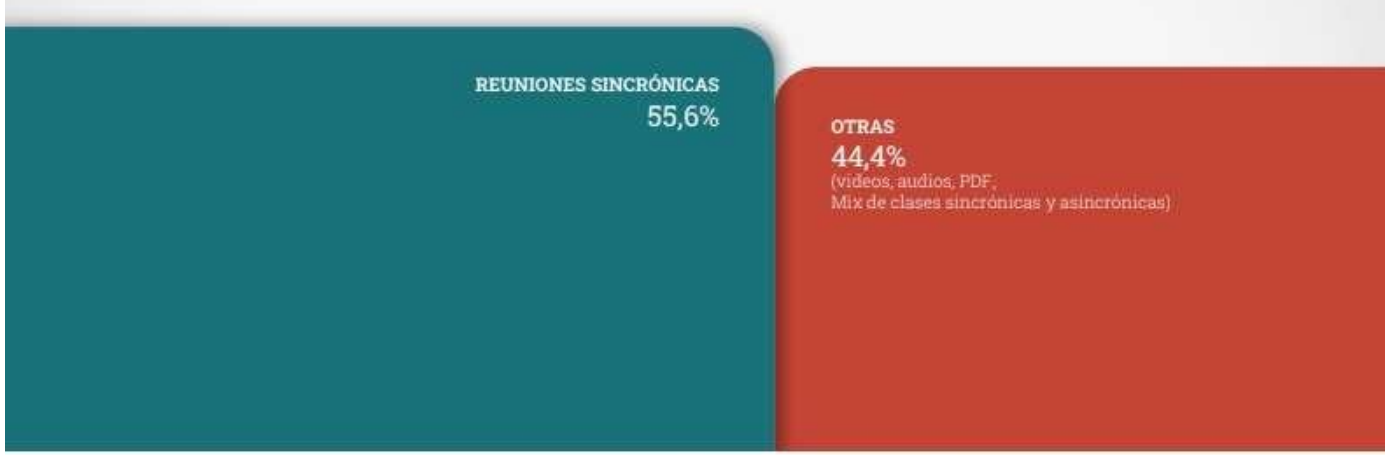

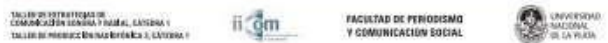


Question/Cuestión, Vol.3, № 70

Diciembre 2021

ISSN 1669-6581
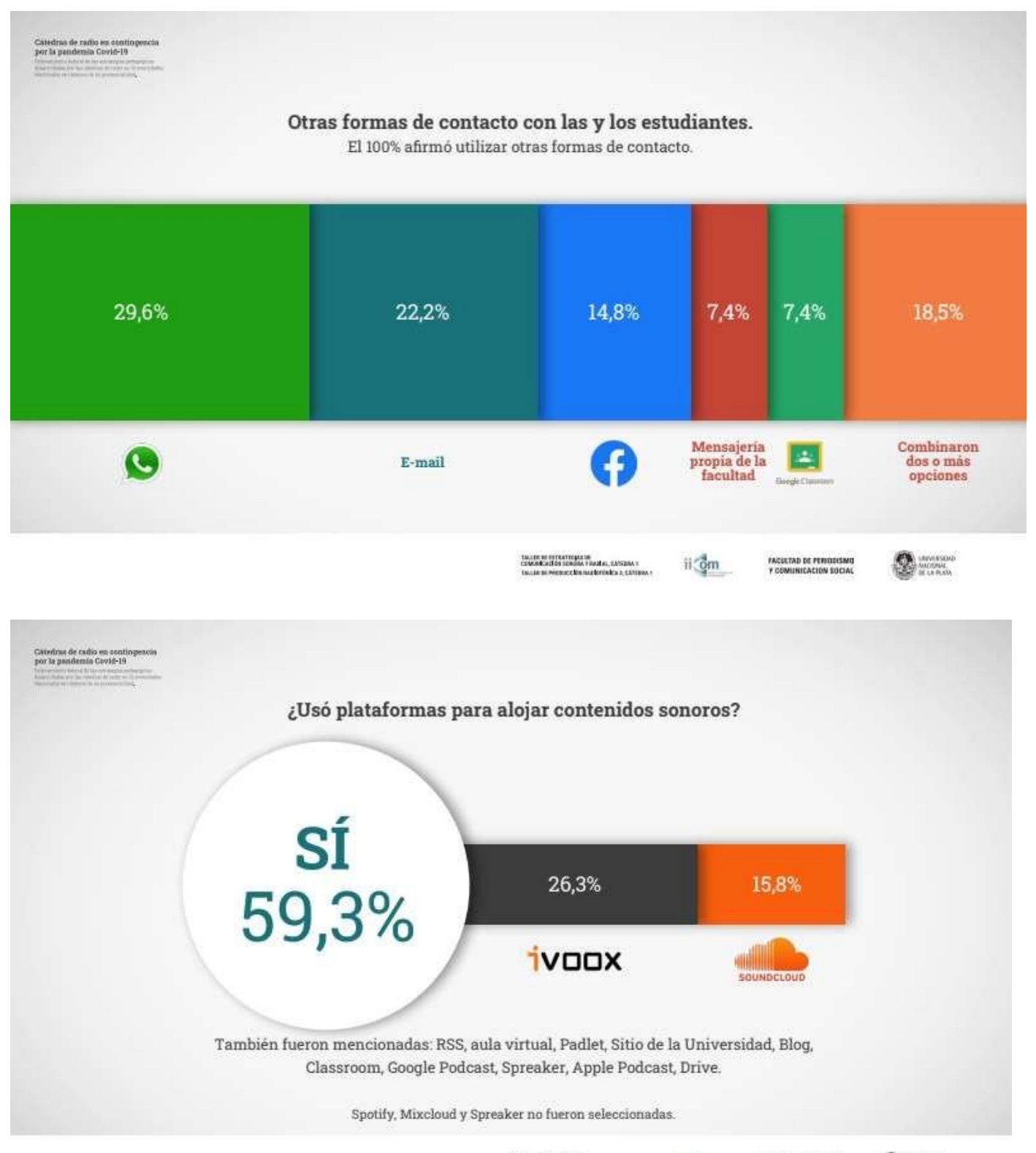

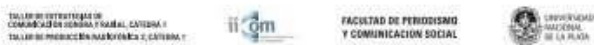


Question/Cuestión, Vol.3, № 70

Diciembre 2021

ISSN 1669-6581
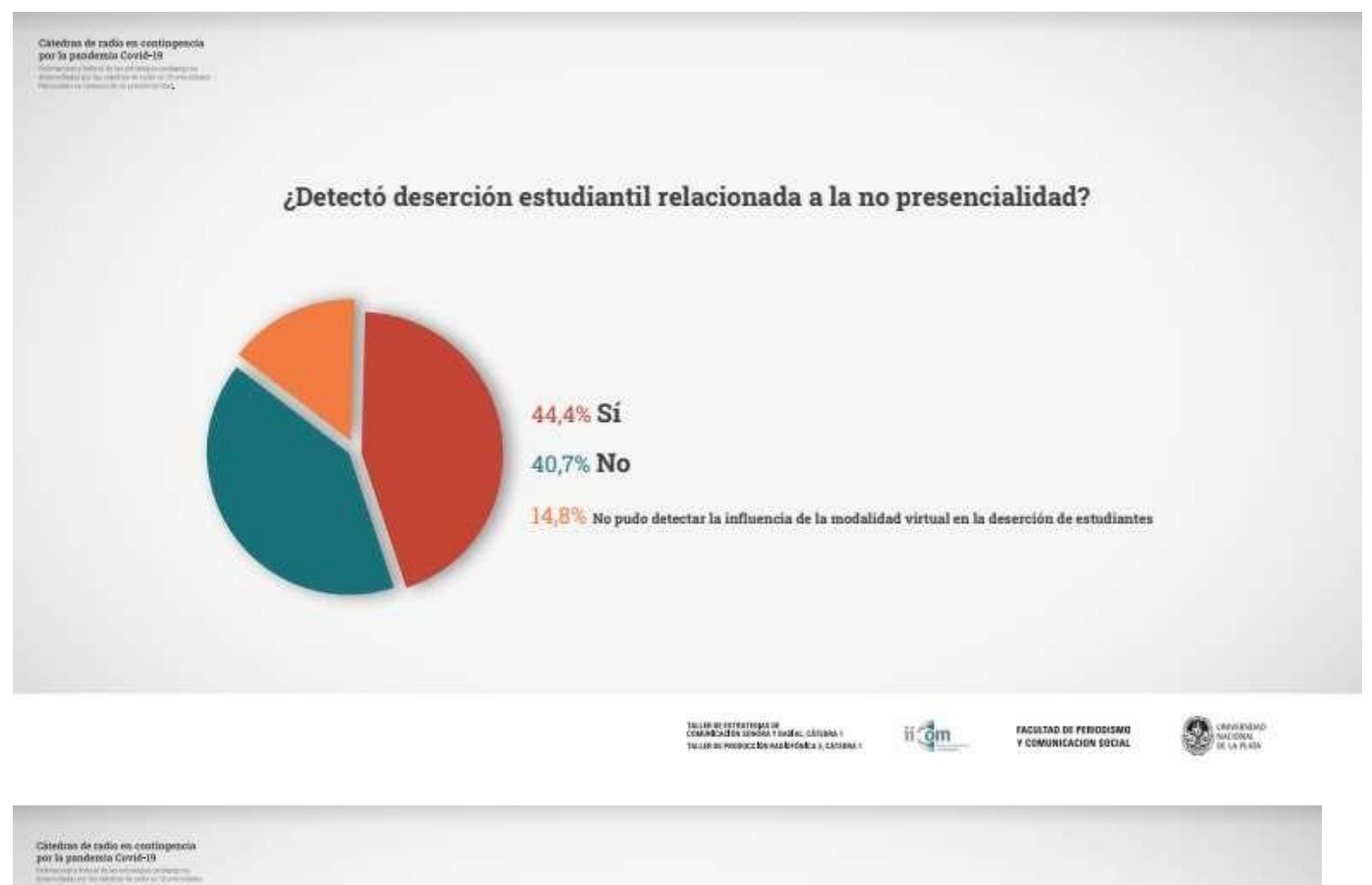

Avance relevamiento federal de cátedras de radio en pandemia.

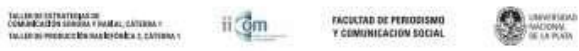


Question/Cuestión, Vol.3, № 70

Diciembre 2021

ISSN 1669-6581

\section{¡MUCHAS GRACIAS!}

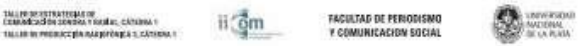

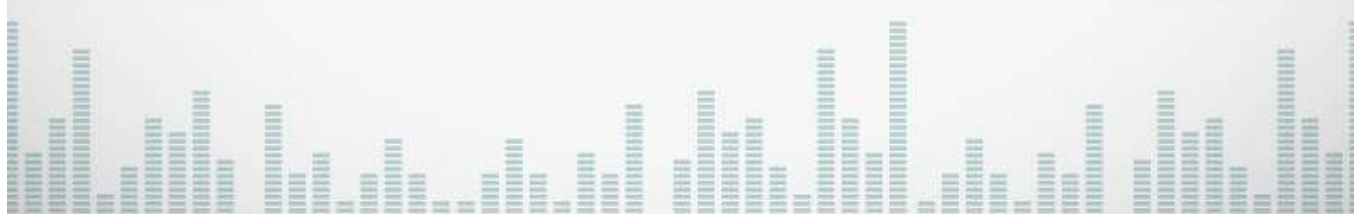

